\title{
La Décentralisation au Mali : État des Lieux
}

Bréhima Kassibo

\section{OpenEdition}

Journals

Édition électronique

URL : http://journals.openedition.org/apad/579

DOI : 10.4000/apad.579

ISSN : 1950-6929

Éditeur

LIT Verlag

Édition imprimée

Date de publication : 1 décembre 1997

\section{Référence électronique}

Bréhima Kassibo, «La Décentralisation au Mali : État des Lieux », Bulletin de l'APAD [En ligne], 14 | 1997, mis en ligne le 26 janvier 2007, consulté le 07 septembre 2020. URL : http://journals.openedition.org/ apad/579 ; DOI : https://doi.org/10.4000/apad.579

Ce document a été généré automatiquement le 7 septembre 2020

Bulletin de I'APAD 


\title{
La Décentralisation au Mali : État des Lieux
}

\author{
Bréhima Kassibo
}

1 Les articles de B. Béridogo, S. Koné, Y.F. Koné et B. Kassibo ont été écrits dans le cadre du GREDEF (Groupe de recherche sur l'État, la décentralisation et le foncier) financé par AIRE-Développement.

2 Si le concept de décentralisation a une longue histoire au Mali, une certaine ambiguïté recouvre le terme, suivant son usage par les différents protagonistes. Le changement politique violent qui s'est opéré en 1991 a provoqué le renversement du régime monopartiste de la seconde république et abouti à l'instauration de ce qu'il a été convenu d'appeler la "démocratie multipartisane". Dès son arrivée au pouvoir, le nouveau pouvoir transitoire incarné par le Comité de transition pour le salut du peuple (CTSP), s'est attelé à jeter les bases de la grande réforme administrative, dont la décentralisation constituait le principal enjeu. La troisième république qui lui a succédé, et dont les fondements étaient fragilisés à l'extrême par une série de facteurs préoccupants: rébellion touarègue dans le septentrion, contestations corporatistes, frondes estudiantines, revendications paysannes, en fit son principal cheval de bataille pour la résolution de certains de ces problèmes, mais aussi, pour mieux consolider idéologiquement son assise politique. Le nouveau pouvoir énonce sa rupture d'avec les précédents (Ie et IIe républiques) considérés comme héritiers du système colonial, et jugés prédateurs et autoritaires. Il prétend réaliser le partage équitable des biens et richesses du pays entre tous ses fils, au nom de la solidarité et de la justice sociale. La décentralisation est présentée dans ce contexte comme l'instrument idéal de réalisation de cette politique dans une perspective de redistribution du pouvoir.

Il s'attelle donc à la création et au renforcement du principal organe d'exécution de cette tâche - la Mission de décentralisation - en vue de l'implantation du processus.

4 Actuellement l'installation de la décentralisation - malgré le retard accusé dans l'exécution du calendrier électoral - a fait du chemin et la manière dont le processus a été mené apparaît pour le moins originale dans la sous-région. En quoi le cas malien se distingue-t-il des autres? Quelles en sont les contraintes (problèmes rencontrés, 
faiblesses notoires) et les perspectives (atouts majeurs et solutions envisagées). Tout ceci constitue autant de questionnements que nous tâcherons d'élucider dans ce numéro consacré à des études de cas, centrées sur les actions menées lors de la première phase de la mise en œuvre du processus au Mali.

Historique

5 Le Mali précolonial, qui a vu se succéder un nombre impressionnant d'empires et de royaumes, a été le théâtre de l'émergence de l'Etat sous diverses. En effet les Etats médiévaux de Ghana, Mali, Songhaï, ont mis au point, au fil des siècles, des systèmes politiques très élaborés, qui ont fait leurs preuves en matière d'administration des communautés et de leurs territoires.

6 La colonisation française s'est greffée sur l'ossature de ces anciennes organisations territoriales : province ou région (jamana), canton (marabolo ou kafo), village (dugu), du moins dans ce qu'on peut qualifier d'anciennes aires culturelles marka, mandé, songhaï et peule. La politique des races, appliquée par l'administration coloniale fiançai se au lendemain de la "pacification" du pays, s'est orientée vers la promotion des leaders politiques traditionnels auxquels elle octroyait le droit d'administrer leurs circonscriptions en tant qu'auxiliaires désignés, au détriment des opposants politiques locaux. Il en a résulté un renforcement des prérogatives de ces nouveaux chefs, que la coutume ne leur reconnaissait point et qu'elle s'était toujours évertuée à contrôler à l'aide d'organes de régulation appropriés (conseils de village, d'arbitrage, de conciliation). Ainsi, les populations furent-elles intégrées dans un vaste système coercitif (travaux forcés, conscription, brimades) dont l'exécution reposait principalement sur la chefferie cantonale. Juché au sommet de cette pyramide le commandant blanc de cercle ou de subdivision demeurait l'épicentre des prises de décisions capitales. Il ajoutait à ces prérogatives administratives, celles judiciaires et de police, signes évidents de son omnipotence arbitraire. C'est cette organisation coercitive de l'administration coloniale qui s'est incrustée dans la mémoire collective et a estompé dans les esprits le souvenir des systèmes d'administration précoloniaux dont certains faisaient preuve de plus de souplesse dans leur application.

7 Dès lors, ceux qui affirment que la décentralisation a véritablement existé au Mali durant la période précoloniale, ne font qu'occulter le véritable débat. Les entités territoriales précoloniales du pays ont été successivement soumises au fil de l'histoire à des formes de domination variées suivant la nature des Etats et celle des liens qui les unissaient au pouvoir central. Elles pouvaient donc temporairement jouir d'une relative autonomie de gestion, ou au contraire subir une dépendance totale en matière d'administration ou même parfois se situer en dehors des aires d'influence de ces systèmes par le hasard de l'histoire, le biais des alliances politiques ou matrimoniales, ou à cause de considérations géopolitiques complexes. Le substrat le plus vivace et récent qui est demeuré, est le modèle colonial dont les Etats post-coloniaux ont hérité et qu'ils ont appliqué sans discontinuer pendant plus de trois décennies. En outre, le vote démocratique qui détermine le choix des responsables des entités territoriales décentralisées, n'est pratiquement connu dans aucune des formes antérieurement évoquées.

8 C'est en ce sens que la décentralisation actuelle est différente des formes antérieures, puisqu'elle instaure le pouvoir de contrôle et de sanction sur les administrateurs grâce au système électif. 
9 La politique d'assimilation forcée des indigènes à la culture coloniale, et l'évolution de la politique française devaient atténuer progressivement les rigueurs de l'administration jacobine, qui s'orienta vers la création de communes indigènes regroupant des citoyens de première et de seconde zones ${ }^{1}$ et bénéficiant pour certaines d'une autonomie relative. Durant toute la période coloniale seules 13 communes furent créées au Soudan français ${ }^{2}$; elles étaient hiérarchisées en communes mixtes, de moyen exercice et de plein exercice, suivant la loi n55-1489 du 18 novembre 1955 . En outre cette municipalisation inachevée ne concernait que le milieu urbain.

10 A l'indépendance, le Mali hérita de cette situation administrative avec cinq communes de plein exercice.

11 Le souci des nouvelles autorités maliennes fut de restaurer la pyramide administrative sur de nouvelles bases. Dès 1958, le parti de l'Union Soudanaise-Rassemblement Démocratique Africain (US-RDA), obtint la suppression des cantons qui étaient dirigés par les chefs traditionnels regroupés sous la bannière du Parti Soudanais Progressiste (PSP), et alliés à la puissance coloniale française.

12 L'article 41 de la Constitution du 22 septembre 1960 énonce déjà la nouvelle structuration territoriale du pays: "Les collectivités territoriales de la République du Mali sont: les régions, les cercles, les arrondissements, les tribus nomades, les communes, les villages et les fractions nomades". Les subdivisions administratives devenaient ainsi des collectivités territoriales qui s'administrent librement par des conseils élus, placés sous la tutelle de l'administration centrale. A l'exception des conseils de communes déjà existant, ces dispositions n'ont jamais été effectives. En fait ces collectivités sont restées des circonscriptions administratives placées sous l'autorité des représentants de l'Etat central.

13 La loi n66-9/AN-RM du 2 mars 1966 portant code municipal au Mali met un terme à la hiérarchisation coloniale précédente en uniformisant le statut des 13 communes sous le régime de plein exercice, mais elle ne donne pas lieu à d'autres créations de communes.

14 Cette situation peut s'expliquer par la politique d'Etat-nation préconisée par les dirigeants de la première république, soucieux de maintenir le peuple dans le carcan de l'unanimisme politique, le parti unique devant être le creuset de l'unité nationale. Le centralisme démocratique qui est de règle, impose la primauté du politique dans tous les domaines de la vie sociale et instaure le centralisme administratif comme instrument d'exécution de cette politique.

15 La première république procède à un renouvellement de l'élite politico-administrative en réaction à la politique coloniale des races qui avait privilégié les leaders traditionnels. Les responsables administratifs deviennent des fonctionnaires nommés par l'Etat selon d'autres critères : compétences professionnelles, engagement politique, etc., soumis à l'autorité de leurs supérieurs hiérarchiques. La naissance, l'héritage et la collaboration ne déterminent plus l'accès à l'exercice de l'autorité administrative depuis la révocation du cantonat. Les chefs de village et de fraction sont réduits au rôle de simples auxiliaires de l'administration dans des activités de collecte d'impôts et à travers les fonctions de représentants du droit coutumier.

16 Le pouvoir s'exerce donc verticalement du sommet à la base de la pyramide et l'uniformisation des pratiques devient la règle générale. En ce sens, la première république est demeurée la digne héritière du système colonial dont le jacobinisme est demeuré la forme privilégiée d'administration, d'où sa qualification par un ancien 
ministre du Développement Rural de la troisième république de "structure tunnel 3" dont la disparition est une des conditions essentielles à la réussite de la décentralisation, dont la restructuration du développement rural préfigure l'avènement.

Le régime militaire issu du coup d'Etat de novembre 68, a dissout les conseils municipaux existants auxquels il a substitué des délégations spéciales nommées par décret. Il procéda néanmoins à un nouveau redécoupage administratif, suivant l'ordonnance 77-44/CMLN du 12 juillet 1977 du Comité militaire de libération nationale (CMLN). La ville de Bamako fut érigée en district subdivisé en six communes ayant à l'instar de celles existantes, le statut de collectivités décentralisées. Cependant, le district demeure une circonscription administrative assimilée à une région et dirigé par un gouverneur nommé par les pouvoirs publics. La même ordonnance laisse la possibilité à l'arrondissement de s'ériger en commune, bien que cela soit devenu un vœu pieux par la force des choses: le pouvoir militaire étant dictatorial dans son essence. Sous la Constitution du 2 juin 1974, en plus de la création du district et de ses six quartiers érigés en commune on a enregistré sur l'ensemble du territoire, la création de la seule commune de Bougouni.

On assiste cependant à la mise en place d'un vaste programme de développement participatif dit "à la base" visant à associer les cadres techniques et administratifs ainsi que les populations locales à la conception et à la réalisation des programmes de développement à travers des organes que sont les conseils et comités de développement. Un fonds de développement régional et local (FDRL), issu de la perception de taxes et impôts divers sert à financer les programmes régionaux et locaux de développement. Cependant, face à l'élite politique locale, l'administration est demeurée maîtresse du jeu en contrôlant et en canalisant l'utilisation de ce fonds vers des actions qu'elle jugeait prioritaires ${ }^{4}$.

19 En fait, la réforme de 1977 a abouti à une déconcentration très poussée de l'administration d'Etat assimilable à une planification du développement, en direction des circonscriptions (région, cercle, arrondissement), sans pour autant réaliser la décentralisation qui avait été préconisée comme l'objectif ultime. Le milieu rural a complètement échappé à la décentralisation.

20 Après mars 91, la "transition" a créé 5 nouvelles communes qui sont : Banamba, Diö̈la, Bandiangara, Niono et Djenné. Avant l'avènement de la décentralisation au Mali, préconisé par la Constitution issue de la Conférence nationale, qui stipule en son titre XI que "les Collectivités Territoriales sont créées et administrées dans les conditions définies par la loi et qu'elles s'administrent librement par des Conseils élus", le Mali ne comptait que 19 communes urbaines. Ce qui a fait dire à un ancien ministre de la transition chargé des réformes institutionnelles, que c'était "le nombre le plus bas du monde pour des bâtisseurs d'empires" (Fofana 1993).

La mise en œuvre du processus de décentralisationLes outils

21 Le Mali a adopté une démarche originale dans le choix de l'approche, en créant une structure souple, appelée Mission de décentralisation (MDD), avec un mandat bien déterminé et une durée d'existence limitée ${ }^{5}$. Cette structure fut dans un premier temps, rattaché au ministère chargé de l'administration territoriale, puis aux services de la Primature, avec mission de concevoir et mettre en œuvre une politique de décentralisation qui mettrait en synergie l'Etat, les collectivités et les représentants de la société civile. D'où le rôle de catalyseur assigné à la Mission chargée d'élaborer les 
instruments juridiques, réglementaires et législatifs indispensables, de concert avec l'ensemble des structures concernées. Un groupe de référence, constitué d'une quinzaine de membres environ sert d'interface à la Mission et aussi de structure de concertation pour la détermination des axes de travail et la validation des options. Dès son rattachement à la Primature, la Mission s'est attelée à une série d'actions orientés vers la mise en place d'un vaste programme de concertation nationale sur les objectifs de la décentralisation et sa pertinence, l'élaboration de textes-cadres en matière de législation et réglementation; et la mobilisation de toutes les forces vives de la nation pour une réflexion sur l'élaboration et la mise en œuvre du processus de décentralisation. Ce qui constitue en somme une approche novatrice dans la sous-région en matière de décentralisation. Dans cette première phase, les Groupes d'étude et de mobilisation aux niveaux régional et local (GREM et GLEM) en tant qu'instruments techniques de la décentralisation, ont été les supports matériels en matière de communication. Ils sont constitués de groupe informels d'hommes et de femmes servant de relais à la Mission à l'intérieur du pays pour l'information et la mobilisation des masses autour des objectifs de la décentralisation.

Le mandat de la Mission consiste à assister le gouvernement dans le domaine de la décentralisation ainsi que dans la conception et la mise en œuvre des mesures nécessaires à cette politique (décret $\mathrm{n}^{\circ} 92-008 / \mathrm{PM}-\mathrm{RM}$ ). Son organisation et ses modalités de fonctionnement sont fixées par l'arrêté n 93-0965/MATS-CAB du 02 mars 1993. Son organisation interne repose sur une typologie par produit et son organigramme repose sur quatre unités fonctionnelles qui sont :

- une cellule chargée de la création des collectivités,

- une cellule chargée du développement des collectivités,

- une cellule chargée du découpage territorial,

- une cellule chargée de la formation des élus.

Ces unités ont été chargées de la réalisation d'un certain nombre de tâches spécifiées par le décret n 93-00I/PM-RM, notamment :

- la définition des rapports entre l'Etat et les collectivités territoriales,

- la conception d'un principe de répartition des biens, des ressources et des charges entre

l'Etat et les collectivités territoriales,

- la proposition de réorganisation territoriale,

- l'élaboration des mesures nécessaires à la politique de décentralisation telle que prévue par

le programme à moyen terme du gouvernement 1992-95.

Après l'identification des objectifs à atteindre et la formulation des produits et activités qui en découlent, la Mission a procédé à une vaste campagne nationale d'information et de mobilisation de tous les acteurs, à travers ses structures techniques que sont les GREM et GLEM; puis elle a procédé à l'élaboration des textes législatifs et réglementaires. Elle a bénéficiée du concours d'experts nationaux et internationaux et de l'appui des partenaires au développement.

Les acquis

Depuis sa création, la Mission a réalisé une grande partie des objectifs assignés dans plusieurs domaines. En ce qui concerne les questions institutionnelles, un cadre institutionnel assorti d'une batterie de lois a vu le jour, notamment :

- La loi n93-008/PM-RM du 11 février 1993 déterminant les conditions de la libre

administration des collectivités territoriales qui peut être considérée comme un texte-cadre, puisque résultant directement de la Constitution (cf. supra). A ce jour, avant la mise en œuvre 
- non encore effective - des collectivités décentralisées, les circonscriptions déconcentrées de l'Etat considérées comme échelons administratifs sont : le district de Bamako, les huit régions, les 49 cercles et les 287 arrondissements. La loi reconnaît comme collectivités territoriales, les régions, le district de Bamako, les cercles, les communes urbaines et rurales qui constituent la base de la pyramide. Il y a trois niveaux d'autorité décentralisée : les régions qui se subdivisent en cercles, les cercles en communes urbaines ou rurales et les communes en quartiers pour les premières et en villages ou fractions pour les secondes, l'arrondissement disparait. Cependant le principe de subsidiarité existe entre les différents échelons. La stratégie de réorganisation territoriale adoptée par la Mission s'est prononcée sur la constitution des communes par suite de regroupements volontaires de villages et de fractions, mais à partir de critères définis. Ce qui a constitué en soi une certaine originalité que certains qualifient de voie malienne de la décentralisation. "Construire la pyramide administrative du pays en partant de bas en haut et en se fondant principalement sur une participation efficiente des populations à la mise en œuvre de la réforme, tel est, au fond, le défi premier qui caractérise ce qu'on pourrait désormais appeler la voie malienne de la décentralisation" (Diallo 1996). La loi supprime le principe de subordination hiérarchique entre les collectivités décentralisées, et attribue un droit de tutelle exercé par l'Etat dans des conditions bien définies. La même loi dote les collectivités territoriales de ressources financières et humaines ainsi que d'équipements et de compétences d'où le transfert par l'Etat des compétences et des ressources.

- La loi W95-034/PM-RM du 12 avril 1995 portant code des collectivités territoriales. Cette loi définit le ressort des affaires locales et détermine les matières à décentraliser en termes de compétence et ressources au niveau des collectivités décentralisées.

- La loi N95-022/PMRM du 30 mars 1995 portant statut des fonctionnaires des collectivités territoriales. Les collectivités s'administrent par deux types de personnel, élus et nommés, mais la loi garantit le statut des fonctionnaires territoriaux par l'uniformisation des situations.

- Le décret n95-210P-RM du 30 mai 1995 déterminant les conditions de nomination et les attributions des représentants de l'Etat au niveau des collectivités territoriales. Il précise la tutelle de l'Etat, représenté au niveau régional par un haut commissaire nommé par décret pris en conseil des ministres. Au niveau du cercle et de la commune il est représenté par les délégués du gouvernement nommés par arrêté ministériel. Les représentants de l'Etat veillent à la sauvegarde de l'intérêt général et assurent une fonction de contrôle et d'arbitrage sur les collectivités décentralisées. Il existe un rapport de subordination entre les représentants du pouvoir central au niveau des circonscriptions administratives, qui s'exprime du haut vers le bas de la pyramide.

- Le décret n 96-084/P-RM du 20 mars 1996 déterminant les conditions et les modalités de mise à la disposition des collectivités décentralisées des services déconcentrées de l'Etat. Il fixe les conditions d'assistance par l'Etat des collectivités, qui se traduit par des prestations temporaires sous forme d'appui, conseil et autres.

- La loi 96-50/PM-RM du 16 octobre 1996 portant principes de constitution et de gestion du domaine des collectivités territoriales. Cette loi reconnaît deux catégories de domaines aux collectivités, l'un privé et l'autre public, dont elle fixe la composante et énonce leur responsabilité dans la gestion, l'aménagement, la conservation et la sauvegarde de l'équilibre de leur patrimoine.

- La loi 96-051 du 16 octobre 1996 déterminant les ressources fiscales des communes. À l'exception des communes du district de Bamako, elle s'applique à toutes les autres. Elle 
détermine la nature des ressources fiscales des communes ainsi que les sources de financement.

- La loi n96-059 du 04 novembre 1996 portant création de communes. Elle parachève la réorganisation territoriale conduite par la Mission, sous le terme de découpage territorial, qui a aboutit à la création de 701 communes. Elle a été votée par l'Assemblée nationale après plusieurs amendements portant sur la suppression ou la création de communes et le transfert de certains villages d'une commune à une autre, sur la base de la liste fournie par la Mission après l'intervention des commissions d'arbitrage et de conciliation. Bien d'autres ont suivi... (cf. bibliographie). Elle procède actuellement à la mise en place d'un nouveau système de communication et de mobilisation pour la seconde phase du processus de décentralisation, et elle a déjà entamé la mise en place d'une banque de données sur les collectivités. Elle procède aussi à la conception de cadres juridiques adéquats de transfert des fonctions, des compétences et de dévolution des biens entre l'Etat et les collectivités territoriales en matière de gestion domaniale et foncière.

uellement la conception et la mise en place de la première phase du processus de décentralisation sont presque achevées mais les élections municipales prévues pour juillet 97 n'ont pu se tenir à cause de la crise politique qui secoue le pays depuis les présidentielles de mai 97. Le mandat de la Mission pourrait se pérenniser, dans l'éventualité (qui n'est pas à exclure) d'une nouvelle prolongation.

Analyse du processus

e relatif succès constaté dans la mise en place du processus de la décentralisation au Mali, a conduit le personnel de la Mission à faire preuve d'optimisme sur l'avenir du processus. Cela se justifie dans la mesure où les cataclysmes annoncés au départ par bon nombre de concitoyens - conflits ethniques, éclatement de l'unité nationale, affaiblissement du pouvoir central, accentuation du régionalisme et résurgence de conflits identitaires, etc. -, ne se sont pas produits, les choses s'étant passées dans un calme relatif. Cependant un bilan provisoire doit être fait, afin d'évaluer le chemin parcouru, mais aussi de dégager les perspectives en ce qui concerne l'avenir du processus. Pour ce faire il y a lieu de passer en revue les phases cruciales qui ont marqué la mise en place des principaux jalons de la décentralisation.

Les politiques institutionnelles

La troisième république a l'instar des deux précédentes a investi l'arène politique avec un discours nouveau, prônant le changement et le mieux être des citoyens. En 1968, lors du coup d'Etat qui a mis fin au régime socialiste de Modibo Keïta, les militaires ont fait irruption par effraction sur la scène politique, au nom de la liberté et de la fin de la dictature marxiste. 23 ans plus tard le régime de Moussa Traoré, rendait gorge sous la pression du peuple appuyé par l'armée. Le mouvement insurrectionnel de mars 1991 fut à son tour porteur d'un nouveau message relatif au bè jè fanga (littéralement: le pouvoir populaire), qui réclamait le partage égalitaire du pouvoir entre tous les citoyens. La dérive anarchique que cela engendra, et qui affaiblit considérablement les fondements de l'Etat, amena celui-ci à restaurer péniblement son autorité sérieusement entamée. De la notion de pouvoir populaire (bè jè fanga), on en vint à celle de pouvoir consensuel (jèèmu fanga) puis à celle du pouvoir négocié (bè ya) pour qualifier le régime démocratique. La troisième république sous la pression des ruraux et du conflit identitaire $\mathrm{du}$ Nord qui contenait en germe la partition du pays, opta pour la 
décentralisation préconisée par la Constitution comme garantissant le principe de la libre administration des populations. La décentralisation considérée comme le transfert de certaines prérogatives de l'Etat vers les communautés de base, servit de support idéologique à la politique de désengagement institutionnel, conseillée par les institutions financières internationales et appuyée par les partenaires au développement. Ce que semble reconnaître le premier magistrat du pays quand il affirme que : "Dans la mesure où nous demeurons une nation essentiellement rurale, je crois pouvoir dire que pour le Mali, la dernière grande réforme de cette fin de siècle est la décentralisation qui demeure la plus grande justification politique de la démocratisation" (Konaré 1996).

Le débat d'idées

30 Cependant, malgré les aspirations d'un peuple écrasé par une dictature de 23 ans, la décentralisation a-t-elle été réellement réclamée comme telle par lui, en toute lucidité? En son temps, la réflexion nationale s'est penchée sur le problème de l'opportunité de cette réforme administrative au Mali. Pour certains caciques de l'Etat ${ }^{6}$ : "La décentralisation est-elle une revendication des populations concernées, c'est-à-dire une réclamation de celles-ci ou s'agit-il d'une vision de ceux qui, après constat des échecs d'une façon d'administrer en déduisent une erreur d'approche à corriger? La dernière alternative qui semble la plus probable porte en elle les germes d'un nouvel échec. Pourquoi ?" (Coulibaly 1993 : 12).

31 L'auteur démontre à l'appui de sa thèse que l'utilisateur de l'outil qu'on veut tailler à sa mesure n'est pas prêt à l'endosser tant sur le plan affectif que cognitif. Même si l'administration décide une fois de plus de ce qui est bon ou mauvais pour les populations et en l'occurrence en projetant un transfert de pouvoir et de compétence au niveau local, nous demeurons, affirme-t-il, dans la même logique qui mène à l'impasse. La décision poursuit-il est toujours verticale à l'origine et ce n'est pas en développant des connexions horizontales de concertation et d'implication supposées que la marchandise devient plus crédible. Et pour lui, la "volonté autogestionnaire des populations ne se décrète pas ; elle prend racine dans leur réalité à la fois économique, politique, sociale, culturelle et parfois psychique". Les populations devraient d'abord exprimer le besoin de la décentralisation. Coulibaly affirme que l'outil décentralisation a servi d'alternative au "statut particulier" des régions du Nord réclamé par les insurgés du Nord afin d'éviter la partition du pays, mais qu'il ne faudrait pas distribuer automatiquement les tickets décentralisation à ceux qui n'en ont point exprimé le besoin, c'est-à-dire, les autres régions du pays qui n'auraient ni la conscience, ni les moyens ni les réflexes pour le faire. Pour lui, chaque communauté doit s'assumer, mais ont-elles les ressources humaines pour s'auto gérer? Car le talon d'Achille de la décentralisation est l'organisation budgétaire conséquente des collectivités, qui exige un arsenal juridique adapté, la détermination du statut des collectivités, les règles de fonctionnement, la gestion du personnel et son statut, celle du patrimoine, etc. D'où il faudrait faire preuve de prudence dans la marche du processus, et amorcer une lecture prudente axée sur une démarche logique, scientifique pour éviter la faillite d'un Etat faible qui par principe ne devrait pas décentraliser...

Dans ce vaste débat qui a mobilisé les consciences au Mali, d'autres voix se sont élevées pour émettre un avis différent. Parmi elles, encore celle du président de la République qui dans un de ces discours, affirmait ceci : " Pour moi, le meilleur passeport pour son entrée [Mali] dans le troisième millénaire, se trouve être le vaste chantier de la 
décentralisation qu'en 1992, j'ai inscrit au rang des priorités au même titre que le règlement du conflit du Nord..." (Konaré 1996).

Le réaménagement territorial

33 En 1996 intervenait la réorganisation territoriale qui a donné naissance aux 701 communes rurales et urbaines du Mali. Dans un cahier spécial encarté dans le journal l'Essor Toumani Diallo (1996) nous fait part de ses réflexions. Pour lui la loi sur la décentralisation ne retient pas l'arrondissement comme la base de la pyramide, en ce sens qu'il apparaît comme pôle de commandement par excellence, mais elle lui substitue la commune. La Mission lors de la réorganisation territoriale a opté pour le regroupement volontaire des villages et fractions en commune et rejeté le principe de la simple transformation des arrondissements en communes. Ce qui pour lui a été une preuve d'originalité dans la sous-région ( $c f$ supra). La mise en place progressive par l'Etat des instruments de la décentralisation, notamment la loi 93-08 définissant les conditions de la libre administration des collectivités territoriales, qui règle l'essentiel des problèmes existant et balise la voie pour d'autres à venir, tout en laissant aux acteurs de grandes marges de manœuvre, suivi par la création d'une structure déconcentrée qu'est la Mission de décentralisation, chargée de la conduite du processus constituerait ce qu'on pourrait qualifier de "processus incrémentai radical de réforme" (Diallo 1995 :14). Processus qui se situerait à l'opposé de la méthode synoptique ou globale qui ne serait qu'une déconcentration arbitraire conduite au pas de charge par l'Etat. L'action de communication et de mobilisation entreprise par les outils informels que sont les GREM et les GLEM créés par la Mission aurait permis une synergie d'actions entre l'Etat et la société civile, et favorisé l'appropriation du processus par les principaux acteurs tout en évitant les écueils redoutés.

34 Entre le pessimisme raisonné affiché par les uns et l'optimisme de bon aloi prôné par les autres la vérité se situerait plutôt à mi-chemin. Au crédit des seconds l'on peut affirmer aujourd'hui que l'élaboration du cadre législatif et réglementaire ainsi que la création des principaux instruments nécessaires à la réalisation du processus ont porté leur fruit. En effet les différents textes relatifs à la création des communes, à leur administration et à la gestion de leur patrimoine ont déjà vu le jour et la réorganisation territoriale s'est correctement effectuée. Et l'on peut valablement affirmer que les populations ont répondu à l'appel et participé objectivement au choix de leur destin.

Les enjeux

35 Cependant les vraies questions demeurent toujours posées quant à l'avenir du processus surtout dans la phase opérationnelle qui doit aboutir à la mise en œuvre pratique des entités décentralisées concrétisée par l'élection du personnel des collectivités territoriales et le transfert du pouvoir du sommet à la base. Ce sont ces nouvelles modalités qui serviront de fondement à nos questionnements. Coulibaly (1997: 10) nous fournit dans l'Essor spécial 22 septembre 1997, une analyse pertinente des enjeux de la décentralisation au Mali ; il s'interroge sur la viabilité des communes qui se heurteront à de multiples contraintes de survie. Sur le plan des ressources humaines, les nouvelles communes seraient confrontées à d'énormes difficultés, car si la décentralisation selon l'adage bamanan, marque "le retour du pouvoir au terroir", l'exercice du pouvoir requiert aussi bien la possession d'aptitudes techniques et administratives par les nouvelles équipes dirigeantes que la disponibilité des moyens financiers et matériels conséquents pour répondre aux grandes attentes des 
populations, conditions que peu d'entités territoriales décentralisées seraient en mesure de réunir.

L'on se rappelle que le découpage territorial exigeait des populations le respect de plusieurs critères, dont les cinq principaux sont d'après le manuel de découpage : les critères socioculturels, les critères démographiques, ceux de distance et d'accessibilité pour les chefs-lieux de commune, les critères de viabilité économique et les critères géographiques et spatiaux. Malgré le libre choix des populations, décrété par la loi fondamentale, le processus ne s'est entièrement pas déroulé sans accrocs. Dans le cercle de Youwarou l'érection de certaines communes s'est effectuée dans le non respect des critères retenus (non viabilité économique, faiblesse démographique, absence totale d'infrastructure dans les chefs lieux, etc.; Kassibo 1997). De même dans la région de Sikasso, les cercles de Sikasso et Koutiala ont donné naissance à une multitude de micro-communes (Diallo 1996 ; Koné), tandis que les cercles de Mopti et de Kidal étaient en proie à la naissance de macro-communes sur les plans géographique et démographique mais dont la viabilité économique était loin d'être évidente.

Ensuite, malgré le choix de décisions accordé aux populations rurales, il a fallu bien souvent l'intervention énergique des commissions d'arbitrage pour décider de la constitution définitive des communes, face à l'absence de consensus des protagonistes conviés à se rassembler. Cela a été attribué par certains à un déficit d'information des GREM et GLEM chargés d'informer correctement les populations sur les règles du jeu. Toumani Diallo (1996), à propos du non respect des critères, pense qu'il s'agissait de critères purement indicatifs dans l'esprit de la Mission, mais que sur le terrain "l'indicatif est devenu norme aux mains des équipes de découpage qui auraient dû les adapter aux spécificités du terrain au lieu de les appliquer mécaniquement comme modèle théorique immuable". Il y aurait eu un déficit de communication ayant amené une disparité dans l'appréciation des critères retenus par la Mission. A propos des micro communes il souligne: "En d'autres termes que dire lorsque la micro-commune est le fruit d'une impression, justifiée ou non, de puissance, en particulier de puissance économique? Rien, sinon déplorer le déficit de participation fructueuse des services techniques dans la détermination plus scientifique de la notion de viabilité économique des constructions communales en cours".

D'autre part le découpage n'a pas pris en compte les régimes de propriété foncière, puisqu'ils n'entraient pas dans ses prérogatives. Cependant la gestion des ressources naturelles qui est soumise à une pluralité de normes et de pratiques contradictoires demeure un élément clé dans la répartition des pouvoirs entre les principaux acteurs, elle peut être porteuse de conflits pouvant menacer l'existence même des nouvelles entités décentralisées. Siby (1997) signale la ruée des villageois pour le contrôle de leur terroir dès l'annonce de la décentralisation. Il en trouve la motivation dans la perception par eux des ristournes sur le montant des transactions effectuées lors de saisies résultant d'infractions commises sur leur territoire. Malgré les autorisations délivrées par les services forestiers à certains exploitants, ces derniers sont interdits d'accès sur les sites d'exploitation par les autorités villageoises et les troupeaux accédant sans permission à leurs pâturages sont frappés de fortes amendes. Le litige opposant Niamiga, un village khassonké de la commune de Maréna Diombougou (région de Kayes), à trois villages soninké Tafacirga, Diallané et Gaméra qui dépendent exclusivement de l'exploitation de son terroir est éloquent à plus d'un titre. En effet, lors du découpage territorial ces trois villages ont quitté la commune pour se rallier à 
une commune à composante soninké, relevant d'un autre arrondissement assez éloigné. En guise de représailles, le village de Niamiga projette de leur interdire carrément l'exploitation de son terroir en vertu du principe de la gestion locale des ressources. Puisque, affirment-ils: "La décentralisation veut dire regroupement des villageois par affinités et prise en charge du contrôle de leur terroir par eux-mêmes, nous entendons nous assumer en ne permettant plus à des gens qui ne nous considèrent pas comme leurs proches, d'exploiter nos ressources".

39 Le choix des chefs-lieux de communes a donné lieu à d'âpres affrontements entre les villages et révélé au grand jour les contradictions qui minent le milieu rural que certains se sont toujours évertués à qualifier d'homogène. Ces faits sont à mettre en relation avec la représentation que se faisaient les villageois du chef-lieu de commune qu'ils considéraient comme un pôle de concentration de pouvoirs, à l'instar du chef-lieu d'arrondissement. En effet le souvenir le plus récurrent dans leur mémoire est celui du chef de canton ou d'arrondissement qui représente encore à leur yeux l'administration déconcentrée dans sa forme la plus immanente et omnipotente ( $c f$ supra). Le maire de la commune serait un simple substitut de "baba coumantan" et le village choisi comme chef lieu concentrerait en ses mains les attributs du pouvoir autrement présentés par la Mission sous forme de critères décisifs de choix, et qui lui permettraient d'exercer son autorité sur les autres.

Ce fait a occulté l'importance du vote pour le choix démocratique des élus, et aussi le principe selon lequel le maire de la commune devrait provenir d'un village autre que le chef-lieu de commune, dans un souci de rééquilibrage des pouvoirs au sein de la collectivité décentralisée.

Les enjeux financiers

41 Les collectivités ont à supporter un certain nombre de charges dont les dépenses de fonctionnement, d'équipement et d'investissement. Elles bénéficient en conséquence de prestations financières sous forme de collecte d'impôts et de taxes, devant leur fournir des ressources conséquentes pour supporter les différentes charges. Si l'on se réfère aux communes existantes, elles n'ont jamais pu faire face aux charges qui leur incombaient. Le niveau de recouvrement des taxes est demeuré très bas dans l'ensemble. De même, sous l'effet de la déconcentration instaurée par la deuxième république les taxes de développement régional et local qui devaient servir à la rétribution du personnel contractuel ne rentraient presque jamais, et certains travailleurs (matrone, aide soignant, agent de la coopération) pouvait rester plusieurs mois sans salaire. Ceci est dû à deux causes essentielles. Au Mali, l'impôt instauré depuis la période précoloniale revêtait pour les populations une consonance négative (ni sanga: prix de la vie, ou di sanga: prix de l'hydromel), il symbolisait l'allégeance forcée à un pouvoir étranger. Sous le régime colonial, l'impôt en numéraire se substitua à l'impôt en nature, sa perception s'effectua par la coercition et il en fut de même sous les Ie et IIe républiques. La troisième république procéda à la suppression de l'impôt per capita, mais maintint la perception des taxes. La population mal informée, fit un amalgame entre les deux et en proie à l'incivisme crut devoir se soustraire à tout paiement de quelque nature que ce soit! Ceci entraîna la vive réaction de l'administration. La décentralisation parviendra-t-elle à transformer les mauvais payeurs d'hier en bon contribuables de demain? D'autre part existe un problème de poids concernant le manque de statistiques sur la matière imposable et les biens de la communes qui doivent être identifiés par rapport à ceux de l'Etat. 
42 Le transfert des compétences et des ressources se fera progressivement aux communes suivant des critères déterminés, mais personne n'est en mesure d'affirmer actuellement ce que l'Etat devra transférer exactement et à qui en premier. Ce qui effraie, c'est l'ampleur de la tâche et les capacités financières limitées de l'Etat lui-même. Il est donc à craindre que les communes n'auront pas le même degré de développement, parce que ne disposant pas des mêmes capacités financières ${ }^{7}$.

Les ressources humaines

43 Autant la gestion des ressources financières demeure problématique, autant celle des ressources humaines reste entière. En effet, même l'Etat manque de personnel qualifié dans des domaines clefs tels que l'éducation, la santé, etc. et il doit fournir aux collectivités décentralisées à travers ses structures déconcentrées un appui conseil et des prestations techniques. Les communes ne disposant pas de personnel qualifié, elles devront procéder à sa formation et en assumer les charges financières récurrentes.

La question foncière

Telle que nous l'avons évoquée plus haut, la bonne gestion des ressources naturelles est l'une des clés de voûte de la bonne gouvernance locale.

A la base de la constitution des communes rurales se trouvent les villages ou les tractions qui sont la base réelle de la pyramide sociale. Le terroir villageois est le lieu d'expression des solidarités familiales, lignagères, intra et inter-villageoises qui se manifestent à travers les rapports sociaux et les modalités d'accès à la ressource qui s'agencent sur le registre des droits coutumiers. Le terroir villageois est un espace socialisé, maîtrisé et hiérarchisé suivant un ordonnancement ancestral. Le foncier est affecté à des ayants-droit, reconnus de tous et jouissant d'une autorité légitime sur l'exploitation de la ressource. Les groupes d'appartenance (lignages fondateurs, détenteurs de maîtrise, alliés, étrangers) règlent l'accès à la ressource suivant les droits d'usage, qui se fondent sur un ordre hiérarchique légitimé par la coutume. Le village est constitué d'un certains nombre de familles ou de lignages regroupés sous l'autorité d'un chef. Les chefs de lignage ou de famille étendue désignent les représentants du conseil de village qui l'assistent dans la gestion des affaires courantes. Traditionnellement la chefferie est dévolue suivant le principe de la primauté d'installation. L'aîné du lignage fondateur instaure son autorité sur la communauté villageoise en vertu du principe cité qui est souvent à base sacerdotale. La chefferie peut être aussi acquise par voie de conquête. A côté de la chefferie politique existent les détenteurs de droits de maîtrise : maître d'eau, chef de pâturage, chef de terre, de la brousse, etc. Ces fonctions sont pour la plupart d'ordre sacerdotal, puisque l'autorité est concédée aux détenteurs par les puissances tutélaires (les génies) qui sont les véritables maîtres de la ressource dont ils leur confèrent l'usufruit. Ces droits sont de nature inaliénable et se transmettent à l'intérieur des lignages récipiendaires. Le patrimoine villageois est constitué du terroir villageois, qui à son tour est contrôlé par les groupes de lignages. L'accès au foncier est tributaire de ce mode d'organisation coutumière qui en garantit l'exploitation. La coutume sert de cadre pour le règlement des litiges fonciers et la chefferie est l'entité la mieux informée sur le code domanial traditionnel et les règles de gestion de la ressource.

Le village n'est nulle part reconnu comme unité administrative, il est l'élément constitutif de la commune et est placé sous son autorité. Dans la perspective de la réorganisation territoriale, il garde encore quelques prérogatives en matière de gestion 
des ressources naturelles mais reste subordonné à l'autorité du conseil communal reconnu comme principal centre de décision.

L'Etat reconnaît, à travers la loi 96-050; aux collectivités territoriales un domaine public et un domaine privé dont elles peuvent disposer. Il leur en concède les droits de gestion et de conservation. Cependant il demeure souverain en matière de transfert puisque par simple décret il peut leur retirer la gestion d'une partie de son domaine public naturel ou artificiel. La section II - portant attribution du conseil communal, de la loi ${ }^{\circ} 95-034 /$ portant code des collectivités territoriales - leur donne la possibilité de délibérer entre autre sur la gestion domaniale et foncière et l'acquisition du patrimoine. Cependant, en son art. 17 il leur est demandé de prendre l'avis du ou des conseils de village ou/et de traction ou des chefs de quartier concernés avant de délibérer sur la gestion du domaine public et privé communal et celle des ressources naturelles. Les autorités traditionnelles ont donc leur mot à dire sur la gestion de leur terroir, mais le problème se situe à un autre niveau, car en matière foncière au Mali, l'Etat a procédé à une définition unilatérale des règles du jeu en dehors des autres partenaires. Il se réclame propriétaire de tous ce qui constitue le domaine public en vertu du principe de domanialité. La sécurisation foncière passe par l'enregistrement et la délivrance du titre foncier par les services domaniaux. Les populations n'ont que l'usufruit de leur domaine qui peut leur être retiré à tout moment suivant les impératifs de l'Etat. Si ces principes ont trouvé droit de cité en milieu urbain, ils demeurent pratiquement ignorés en milieu rural où le foncier est conçu comme un patrimoine lignager inaliénable et soumis au droit coutumier. L'appropriation foncière, l'accès aux ressources son soumises à des règles non écrites, les conflits sont réglés par des instances de conciliation et d'arbitrage jouissant d'une légitimité sociale. Le droit positif ne reconnaît pas explicitement le droit coutumier et se donne l'exclusivité en matière d'élaboration de code foncier. Dans la pratique on assiste à une superposition de droits et les différents acteurs jouent sur les différents registres en fonction de leurs intérêts. Si le maire et les conseillers communaux ont pour eux la légitimité des urnes, les ayants-droit locaux jouissent d'une autorité sacerdotale et d'une légitimité sociale. Quel type de légitimité devrait primer en matière de gestion du foncier et de l'accès à la ressource? En fait, il faudrait élaborer un nouveau code foncier qui reconnaîtrait la légitimité des droits coutumiers et associerait les gestionnaires traditionnels à l'élaboration des textes sur le foncier. L'Etat doit donc transférer une partie de ses prérogatives aux instances locales,. pour une nécessaire harmonisation des droits. Or ce que nous observons actuellement c'est le maintien d'un code foncier uniforme, élaboré par l'administration en dehors des instances coutumières, et suivant les principes du droit positif, et dont la relecture annoncée de longue date, tarde à se concrétiser. Nous pensons pour notre part que seule une symbiose axée sur les pratiques locales et réalisée par tous les intervenants du foncier pourrait avoir droit de cité.

La question foncière a beaucoup influencé le choix des populations lors du réaménagement territorial. C'est ainsi que sur les 682 nouvelles communes on ne compte qu'un nombre infime de communes urbaines. Sur 40 agglomérations pouvant prétendre à ce titre, seules 20 s'étaient déclarées et il n'en subsistait que dix-huit aux restitutions régionales. La dynamique des populations s'est orientée vers la constitution de communes rurales dans le souci d'élargir le patrimoine foncier en intégrant les terres des villages satellites, soit pour des raisons de sécurité foncière ou purement spéculatives. Certaines circonscriptions déjà érigées en communes urbaines ont opté pour le statut de communes rurales (ex. de Niono, Dioila, Banamba, Fana, 
Massigui, etc.). Ceci fait craindre une tendance à la transformation des terres agricoles en habitat urbain, eu égard à la spéculation foncière découlant de la marchandisation des terres dont l'exemple est donnée par l'Etat lui-même en tant que principal opérateur économique en matière d'aliénation du patrimoine foncier. En effet l'exemple des communes de Bamako est éloquente à ce égard (Traoré 1997). Non seulement les communes ont progressivement accaparé toutes les terres des villages périphériques à des fins spéculatives, mais à travers l'opération "Sauvons notre quartier" les maires et conseillers des communes I, II, IV, V et VI du district, se sont attribués la majorité des lots de recasement réservés à la population des zones à lotir ${ }^{8}$. (Diallo 1996) attribue ce déficit de communes urbaines à une carence d'information de la part des organes de communication qui auraient porté l'accent sur les communes rurales au détriment des communes urbaines, mais il reconnaît aussi des velléités de spéculations foncières futures au bénéfice des communes.

Le déficit démocratique

Le succès du processus de décentralisation ne dépendra pas que de la Mission de décentralisation qui. l'a mis en marche, mais de son degré d'appropriation par la population malienne. Or plusieurs contraintes doivent être surmontées pour relever ce défi. L'élargissement de l'espace d'expression démocratique nécessite la participation de tous à travers le dialogue et le débat. Un des problèmes clés de la décentralisation a été le déficit d'information des populations sur le sens réel du processus, lié à l'inadéquation du véhicule linguistique. Le terme utilisé en langue française ne colle point au contexte d'un pays dont la majorité ignore cette langue. Il a fallu donc se livrer à l'exercice périlleux de la traduction du concept en langue nationale. C'est ainsi que par la magie de la traduction, la population du Mali profond a appris de la bouche des traditionalistes recrutés par la Mission que le terme "décentralisation" signifiait en langue bamanan "mara ka seki sa" c'est-à-dire le retour de l'administration au terroir. D'où la conclusion que la décentralisation est un phénomène connu des maliens puisqu'ayant existé bien avant la période coloniale qui lui avait substitué l'administration centralisée. Elle devenait donc avec l'avènement de la démocratie multipartisane (bè ya) l'expression du pouvoir consensuel.

50 Même si son application émanait de la volonté du peuple, il n'empêche qu'elle a été portée sur les fonts baptismaux par la volonté du pouvoir politique, et conçue par une équipe d'experts et de technocrates. Le processus de remembrement territorial est venu compliquer un peu plus le problème, car les populations ont été invitées à faire ressusciter les anciennes entités territoriales (cantons ou marabolow), à travers les noms des nouvelles communes. Ceci créa une nouvelle confusion chez les ruraux chez lesquels le retour à l'ordre ancien réveilla les convoitises et l'appétit du pouvoir. L'exemple du choix des chef-lieux de communes en fait foi ${ }^{9}$.

51 L'analyse de la société malienne, laisse entrevoir une dichotomie profonde dans son mode de fonctionnement qui se caractérise par l'opposition ville-campagne. Le mouvement de mars 91 a été_réalisé par des citadins et surtout ceux de la capitale du pays, proches de l'épicentre du pouvoir. À la chute de la IIe république, la revendication fondamentale qui était l'avènement de la démocratie multipartisane une fois satisfaite, le front démocratique se lézarda, en proie aux affrontements pour la conquête du nouveau pouvoir. La capitale apparait comme l'unique centre de décision, soumis au bouillonnement $\mathrm{du}$ microcosme socio-politique. En fait, il s'agit d'un milieu profondément extraverti, où les hommes politiques en proie au mimétisme culturel 
appréhendent la réalité malienne d'un œil différent de celui de la majorité de la population essentiellement formée de ruraux. Ainsi la démocratie malienne dans sa phase transitoire fut portée aux nues par ses serviteurs et devait servir d'exemple au pays de la sous-région. Sous la poussée du monde rural, des bailleurs de fonds, et l'emprise des intellectuels, la décentralisation est devenue donc le passage obligé pour le partage du pouvoir de commandement. Le schéma est indiscutablement issu du modèle occidental inconnu d'une population à majorité analphabète et écrasée sous le poids de plus de 30 ans de dictature. L'application du schéma devrait poser problème dans la mesure où le peuple et son élite ne parlent pas le même langage. L'émergence des principaux organes de renforcement de la démocratie que sont les média, les partis politiques et la société civile, n'a pas radicalement comblé le déficit démocratique. Ces organes faiblement implantés en milieu rural ignorent pour la plupart les réalités du pays profond, et les états majors se livrent à des luttes partisanes au sein du microcosme bamakois, pour la défense d'intérêts corporatistes et la conquête du pouvoir dont l'alternance est devenu synonyme de conflits fratricides.

" obéit à une autre logique axée sur le vécu quotidien. Ecrasé sous le poids de la misère et la ponction de ses ressources par l'élite urbaine, le monde rural a fini par réclamer son dû, c'est-à-dire son affranchissement du carcan de l'Etat prédateur.

Les Etats généraux, tenus en 1991, ont été l'expression la plus radicale des revendications paysannes, qui s'exprimaient sporadiquement par la fronde envers les représentant du pouvoir central. Il est évident que le monde rural voulait une réforme fondamentale du système politico-administratif, la réponse lui fut fournie par l'outil décentralisation taillé à sa mesure et proposé à son assentiment.

Dès lors pour la réussite du processus il y a lieu de lever l'obstacle de la communication et de transformer "les espaces de palabres en espaces d'expression démocratique" (Coulibaly 1997). La société rurale malienne est une société fortement hiérarchisée où chacun retrouve sa place en fonction de son statut fixé dès la naissance mais qui peut varier suivant l'âge et le sexe. La décentralisation en tant que transfert des prérogatives de l'Etat au niveau local induit des changements d'attitudes profonds sur les plans social, économique et politique. Elle provoque un bouleversement des structures anciennes et l'émergence de nouveaux acteurs. Les enjeux se situent à plusieurs niveaux. Seule une bonne politique de communication pourrait lever la contrainte de l'incompréhension, et provoquer une faille dans la muraille du pouvoir traditionnel où certains groupes sont exclus à cause de leur statut marginal. Dans la conception traditionnelle, l'exercice du pouvoir est réservé à des ayants-droit dont la légitimité est reconnue de tous. Ce sont les représentants des lignages fondateurs ou dominants appelés halalaw, yèrè wolow gestionnaires désignés du marabolo ou commune. Face à eux dans la plupart des cas, les sigi n'jèw ou dunanw que sont les étrangers, les nyamakalaw ou hommes de caste, les wolosow ou descendants d'esclaves, les dénw (cadets ou fils) sont écartés définitivement ou temporairement de l'exercice du pouvoir, sans parler des femmes qui sont les éternelles mineures. Le défi démocratique consiste à élargir ces cercles concentriques afin d'y faire accéder le plus grand nombre, en vertu de leur compétence et non uniquement par la naissance ou autres privilèges. En cela, les partis politiques et les masses média doivent contribuer à l'émergence d'une société civile éclairée, empreinte de civisme et du sens des responsabilités : c'est une des conditions indispensables à la réussite d'une décentralisation véritable capable d'intégrer les 
marginaux et de les informer sur leurs droits par rapport à leurs représentants. Ceci correspondrait au second sens attribué à la décentralisation; ka mara kan da bo, i.e., étendre le cercle d'exercice de l'autorité administrative.

Les conditions de l'aide

Dans la mise en place du processus de décentralisation, l'Etat malien a largement bénéficié de l'aide de "pays amis" et des institutions internationales. Mais cette aide est-elle si désintéressée qu'elle le paraît? Pour Félix (1996), l'Etat malien et la nouvelle classe politique voient dans la décentralisation "un instrument de la démocratie" tandis que les institutions de Bretton Woods y voient "un outil au service du marché" et les organismes d'aide au développement "un vecteur de développement local". Concomitamment au processus de décentralisation administrative l'aide aussi prend le chemin de la coopération décentralisée qui devrait se substituer à la coopération bilatérale entre Etats. Le privé et les organisations non gouvernementales apparaissent progressivement comme les relais de distribution de la manne financière et du savoir-faire technique. Une nouvelle structuration du mécanisme de l'aide internationale se met en marche et se nourrit des subsides du marché de l'alimentaire caractérisé par l'aide d'urgence et le devoir d'ingérence humanitaire dans la politique des Etats. La bonne gouvernance, le désengagement de l'Etat à travers la privatisation du secteur public, l'intégration au marché mondial et la décentralisation de l'administration deviennent les critères de choix pour l'éligibilité des gouvernements au statut de récipiendaires privilégiés. Les meilleurs reçoivent primes et encouragements et leurs populations payent au prix fort les efforts consentis pour la réussite du programme d'ajustement structurel de plus en plus renforcé.

La Mission a reçu des "partenaires au développement" une aide financière conséquente, soit :

140 millions FCFA pour les fonds d'étude, 300 millions au titre de l'assistance publique et 120 millions pour la communication, de la part de la CEE; soit un montant total de 560 millions. L'Allemagne à travers la GTZ a alloué 1 milliard de FCFA, au titre de l'assistance technique (programme de formation). Le PNUD a octroyé 250 millions de FCFA au titre de l'assistance technique pour l'aménagement du territoire et le programme de développement. Le FAC a donné 400 millions FCFA au titre de l'assistance au fonctionnement de la Mission et de la préparation des transferts. La Suisse a octroyé 10 millions FCFA pour la communication. Les Pays-Bas ont financé le fonctionnement des GREM à concurrence de 60 millions FCFA. La CFD (Caisse française de développement) a fait don de 50 millions FCFA, pour l'étude sur les ressources des collectivités et sur le foncier. L'USAID a octroyé 30 millions FCFA au titre de l'appui à des actions de formation à la base. Le Canada n'est pas demeuré en reste : il a octroyé 260 millions FCFA pour la création et formation des GLEM et le découpage territorial. Certains partenaires se sont d'ores et déjà prononcés pour financer les élections et le démarrage des communes (CEE, USAID ${ }^{10}$ ). Quand on sait que l'altruisme n'est point de règle en matière d'aide bilatérale, on ne peut que souhaiter que le prix à payer ne soit trop élevé au vu des efforts généreusement consentis. De plus, soumis à l'influence des partenaires différents qui n'ont pas la même approche du processus de décentralisation, à cause de leur référents culturels et historiques ${ }^{11}$, la Mission aura beau jeu de s'en tenir aux réalités de terrain en se soustrayant aux cris de sirène des technocrates et experts es-décentralisation drapés dans leurs subtils manteaux de conseillers techniques. 
Conclusion

$\mathrm{Au}$ bout de trois ans d'existence, la Mission semble avoir gagné la première tranche de son pari, à savoir mettre la décentralisation sur les rails. Néanmoins beaucoup reste encore à faire car le chemin est parsemé d'embûches. En fait le problème qui se pose au peuple malien, c'est de ré-inventer un autre type d'Etat. Ce qui exige de prime abord un changement radical de mentalité, qui ne saurait intervenir sur commande, et une réelle volonté de changement. Il s'agit avant tout de réconcilier l'Etat avec la majorité de la population condamnée à jouer perpétuellement le second rôle par rapport à une élite minoritaire.

Les élections passées ont démontré la perte de confiance de la population envers les politiciens maliens engagés dans des luttes partisanes et fratricides, ce qui se traduit par un très fort taux d'abstention, croissant au fil des élections successives. Bien ambiguë demeure la position de l'administration qui devrait nécessairement effectuer sa mue en passant d'une structure de commandement à une structure de conseil, de développement et d'arbitrage. Il reste à observer son attitude prochaine et celle des élus locaux, à propos du transfert des prérogatives de l'Etat vers les collectivités décentralisées et du nouveau mode de gouvernance locale. Les populations rurales qui constituent la part la plus importante de la société malienne, sont-elles prêtes à assumer leur destin en s'identifiant véritablement au processus pour se l'approprier, afin d'en devenir les véritables acteurs ? Ceci semble évident pour le premier magistrat du pays pour qui : "la décentralisation avant d'être une dynamique économique, est un état d'esprit qui réhabilite le monde rural au triple plan, moral, administratif et politique... et les $702{ }^{12}$ communes sont autant de forums au service du développement et de la démocratie" (Konaré 1997). Gageons qu'il sera entendu...

\section{BIBLIOGRAPHIE}

Coulibaly, M. 1993. "La décentralisation... pour quoi faire ?". Décentralisation, journal d'information et de réflexion sur la décentralisation (février), Mission de Décentralisation (Bamako).

Coulibaly, M. 1997. "Les nouvelles communes sur les fonts baptismaux. Ressources : le temps des vraies interrogations". L'Essor (Bamako, $\mathrm{n}^{\circ}$ spécial du 22 septembre).

Diallo, T.D. 1995. "La décentralisation : Rendre son âme au Mali profond". Le Démocrate Malien (Bamako éd. spéciale, juillet).

Diallo, T.D. 1996. "Le Mali nouveau ; vers une nouvelle géographie régionale". L'Essor (Bamako, 18 juin, cahier spécial décentralisation).

Felix, J. 1996. "L'élaboration de la politique de décentralisation au Mali : des logiques plurielles". Bulletin de l'APAD 11 :148-163.

Fofana, W. 1994. "A propos du projet de loi sur la libre administration des collectivités". Décentralisation, journal d'information et de réflexion sur la décentralisation (février), Mission de Décentralisation (Bamako). 
Kassibo, B. 1997. L'histoire d'un dépeçage ou la naissance controversée des collectivités décentralisées du cercle de Youwarou. Louvain-La-Neuve : Séminaire sur "les dimensions sociales et économiques de développement local et la décentralisation en Afrique au Sud du Sahara" (20-21 nov).

Konare, A.O. 1996. Discours officiel du 22 septembre. Bamako : Archives de l'ORSTOM.

Konare, A.0.1997. Discours officiel du 22 septembre. Bamako : Archives de l'ORSTOM.

Siby, M. 1997. Les populations rurales maliennes el la décentralisation: Etude de cas de la commune de Maréna-Diombougou. Bamako : ENS (mémoire de maîtrise).

Traore, I.S 1997. Pouvoirs locaux et décentralisation au Mali : cas de la commune V du district de Bamako. Bamako : ENS (mémoire de maîtrise).

Mission de Décentralisation, 1996. Lois et décrets de la décentralisation. Bamako : Primature ;-Mission de décentralisation ; Bamako. Ce recueil contient 11 lois et décrets relatifs à la décentralisation malienne qui sont :

La Constitution de la République du Mali du 25/02/92.

1. Loi n93-008 du 11/02/1993 déterminant les conditions de la libre administration des collectivités territoriales.

2. Loi n95-034 du 12/04/1995 portant code des collectivités territoriales en République du Mali.

3. Loi n95-022 du 20/09/05 portant statut des fonctionnaires des collectivités territoriales.

4. Décret n095-210/P-RM du 30/05/95 déterminant les conditions de nomination et les attributions des représentants de l'Etat au niveau des collectivités territoriales.

5. Loi n96-025 du 21/02/96 portant statut particulier du district de Bamako.

6. Décret n96-119/P-RM du 11/04/1996 déterminant les conditions de nomination et les attributions du représentant de l'Etat au niveau du district de Bamako.

7. Décret n96-84/P-RM du 20/03/1996 déterminant les conditions et les modalités de mise à la disposition des collectivités territoriales des services déconcentrés de l'Etat.

8. Loi n96-050 du 16/10/1996 portant principes de constitution et gestion du domaine des collectivités territoriales.

9. Loi n96-051 du 16/10/1996 déterminant les ressources fiscales des communes.

10. Loi n96-058 du 16/10/1996 déterminant les ressources fiscales du district de Bamako et des communes qui le composent.

11. Loi n96-059 du 04/11/1996 portant création de communes. Journal officiel de la République du Mali (spécial n³ du 26 novembre 1996), Bamako : Secrétariat Général du Gouvernement.

Manuels de la Mission de décentralisation 1994. Parmi les plus importants nous pouvons en citer quatre, qui sont :

1. Manuel de formation au découpage territorial ;

2. Guide du découpage territorial ;

3. Stratégie de formation ;

4. Stratégie et Programme d'action.

Dans le cadre de la politique d'information, d'éducation et de communication, plusieurs messages ont été véhiculés sous support audio (cassettes). 
Un atlas a été publié en 1997. Il comporte l'ensemble des communes consacrées par la loi 96-059 du 4 novembre 1996.

Un CD-ROM a aussi été diffusé en 1997. Il contient les informations essentielles sur les 701 communes du Mali.

\section{NOTES}

1.Les communes de Kayes et de Bamako furent créées en 1918 en tant que communes mixtes de premier degré. Celles de Mopti et Ségou furent créées en 1953 et celle de Sikasso en 1954 comme communes mixtes. La loi de 1955, érigea Sikasso et Mopti en communes de plein exercice en 1955 et Sikasso en commune de moyen exercice en 1956, puis de plein exercice en 59. En 1958 huit nouvelles communes de moyen exercice furent créées, il s'agit de: Kita, Nioro, Kati, Koulikoro, Koutiala, San, Tombouctou et Gao. Cest ainsi que certains fonctionnaires noirs de l'Afrique de l'Ouest, dans le but de conférer à leurs progénitures la citoyenneté française envoyaient accoucher leurs épouses dans la commune de Saint-Louis du Sénégal qui bénéficiait d'un statut particulier.

2.Appellation coloniale du Mali actuel.

3.Il s'agit de feu Boubacar Sada Sy, qui a assumé la fonction de ministre du Développement Rural avant d'aller au ministère de la Défense sous la IIIe république. Il disait notamment ceci: "Peu de gens savent encore que nos structures 'tunnel' sont héritées de l'armée. L'administration malienne est calquée sur l'année. C'est l'armée coloniale qui a donné les noyaux des principaux services... Donc, on a un chef à Bamako, et ce chef a ses agents dans un tunnel au plus loin jusqu'où il peut aller. Nous avons hérité de cela, nous l'avons agrandi, nous l'avons entretenu, nous en avons fait des instruments d'intervention de l'Etat. Dans notre plan d'action nous voulons casser cette structure là... Notre plan d'action est une anticipation de la décentralisation". 4.Par exemple, la réalisation des travaux d'infrastructures telles que la construction de la case de passage du président de la république ou du chef de l'exécutif régional donnait lieu à des détournements massifs de fonds par les membres influents des comités de développement.

5.La MDD a été créée par le décret n93-00/1PM-RM du 06 janvier 1993 pour une durée d'un an et rattaché au ministère de l'Administration Territoriale et de la Sécurité, puis prolongée pour une durée de 3 ans, par décret 94-051 du 26 janvier 1994 et rattachée cette fois-ci à la Primature.

6.A cette date il était conseiller à la présidence de la troisième république. 7.En son art.27, ch. 4, la loi Nº3-008/déterminant les conditions de la libre administration des collectivités territoriales dit que "les collectivités territoriales des régions de Tombouctou, Gao et Kidal bénéficient pendant une période de cinq ans de dotations spéciales annuelles versées par l'Etat conformément à l'esprit du "Pacte national".

8.A titre d'exemple, sur les 228 lots proposés à la population de Djénékabougou (Djikoroni Para), plus de la moitié, soit 124 lots ont été accaparés par les membres de la Commission de recasement, les techniciens géomètres chargés du lotissement, le maire de la commune et ses conseillers. Malgré le résultat des enquêtes qui ont établi la véracité des faits, les plus hautes autorités du pays ont été incapables de rétablir la population dans ses droits. C'est dire que la spéculation foncière renforcée sous la IIIe 
république est devenue un moyen de promotion sociale aux mains des élus municipaux, qui accaparent les terrains à bâtir et les revendent aux particuliers nantis, à des prix exorbitants. Ils édifient ainsi des châteaux et roulent en carrosse, jouissant d'une totale impunité, et d'une indulgence coupable de la part des pouvoirs publics au sein desquels ils semblent jouir de complicités tacites.

9.Jusqu'à ce jour, malgré la promulgation de la liste des communes par l'Assemblée nationale, des contestations émanent de certains villages qui réclament de nouveaux arbitrages. Ainsi, un douanier fut lynché et brulé vif par une partie de la population de Yanfolila en révolte contre l'autorité administrative chargée de la répression de la fraude et de la contrebande. Les agents forestiers chargés de la police rurale étaient particulièrement visés par les villageois.

10.United States Agency for International Development.

11.En matière de décentralisation, la conception juridique romano-germanique est radicalement différente de l'anglo-saxonne. A la question de savoir, par exemple, quel est l'élément déterminant entre la société civile et la structure décentralisée qu'il faut promouvoir en premier lieu, la réponse fournie par un anglo-saxon risque d'être fort différente de celle de son homologue latino-germanique.

12.En fait, il en existe officiellement 701, la 702ème étant encore à l'état de projet lors du discours du président de la république.

\section{AUTEUR}

BRÉHIMA KASSIBO

Anthropologue GREDEF, BP 84, Bamako (Mali). Tél. : 223/222774. Fax. : 223/2275 88. 